\title{
The fragmentation of expanding shells - limitations of the thin-shell model
}

\author{
Jim Dale, ${ }^{1}$ Richard Wünsch, ${ }^{1}$ Jan Palouš ${ }^{1}$ and Ant Whitworth ${ }^{2}$ \\ ${ }^{1}$ Astronomical Institute, AVCR, Prague, Czech Republic \\ ${ }^{2}$ School of Physics and Astronomy, Cardiff University, Cardiff, UK
}

\begin{abstract}
We study the fragmentation of expanding shells in the context of the linear thin-shell analysis. We simulate shell fragmentation using the FLASH AMR code and a variant of the Benz SPH code.
\end{abstract}

Keywords. stars: formation, ISM: bubbles

The full poster (in pdf format) is available at

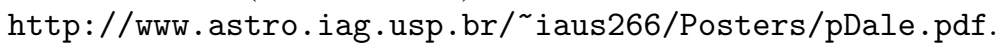

